together to report on the educational and financial value of education plans.

Results The work of the Clinical Support Analyst is enabling standardisation of the data collection. The CPF has collated four months of activity data, manipulating visual representations of the data to analyse his time allocation. Using an electronic booking system gives a streamlined process for the organisers and attendees, making more efficient use of administration time.

Conclusions The work is ongoing, initial conclusions are as follows. The Clinical Support Analyst prompted a formalisation of data requests and rigorous recording of the time work takes him to complete. It has also highlighted that the data from the electronic patient notes does not match the data collected locally. The CPF has found that the App has to be used with rigour to ensure full data capture. Not all members of staff are confident enough to book their own training and do not read their emails in a timely way.

\section{P-55 DEVELOPMENT OF PERSON-CENTRED CARE AT A HOSPICE}

Sarah Wells, Michele Wolfe, Fiona Dawes, Nicky Baker, Suzanne McArthur. Marie Curie Hospice, West Midlands, UK

\subsection{6/bmjspcare-2018-hospiceabs.80}

Background We have had an electronic patient record (SystmOne) at the hospice for the past five years. This is our tool for documenting the care that we provide, viewing and reviewing these notes and reporting on this data. Our patients and families have input from many members of the multi-disciplinary team to ensure that they receive person-centred holistic care. Our electronic patient record, SystmOne was structured so each discipline completed initial assessments within a communal template but ongoing care tended to be written as separate reviews and action plans. The result of this was silos of information and staff not reading each others notes and using the information obtained from patients and families to the optimum. We recognised that the crucial bits of information from patients that make a huge difference to their experience were not being captured and communicated well.

Aims To put people at the centre of their care and support and maximise their involvement through development of person-centred templates on SystmOne.

Methods Multidisciplinary team meetings explored the current methods of documentation. Using patient journeys, a gold standard method of documentation was designed within SystmOne. Generic nursing care plans were removed and replaced with a multi-disciplinary 'About me' Care plan.

Results An 'About me' care plan pops up as soon as an electronic record is opened. It is completed by all members of the MDT and tells us how to care for a patient. It is divided into seven domains of care. It includes phrases like 'Julie likes to have a Baileys in the early evening'. All other elements of care are documented in a person -centred care template by all disciplines.

Conclusions The new method of documentation puts the patient at the centre of their care and ensures their care and support is completely individualised.

\section{P-56 DO ELECTRONIC PALLIATIVE CARE COORDINATION SYSTEMS (EPaCCS) INCREASE THE CHANCES OF A GOOD DEATH?}

${ }^{1}$ Lucy Pocock, ${ }^{1}$ Sarah Purdy, ${ }^{2}$ Michelle Farr, 'Lydia French, ${ }^{1}$ Richard Morris. ${ }^{1}$ University of Bristol, Bristol, UK; ${ }^{2}$ CLAHRC West, Bristol, UK

\subsection{6/bmispcare-2018-hospiceabs.81}

Background People at the end of life frequently receive care from a variety of teams and organisations. Continuity of care is particularly important for patients at the end of life and a lack of information sharing is often cited as a barrier to the provision of good quality end of life care.

Nationally, the policy drive to address this has been the development of electronic palliative care coordination systems (EPaCCS). The purpose of EPaCCS are to provide a shared local record for health and social care professionals, with key information about an individual approaching the end of life, including their expressed preferences for care.

Quantitative studies have shown striking differences in place of death with EPaCCS, but are potentially biased and confounded. Technology in isolation is not guaranteed to bring benefit and the initiation of an EPaCCS relies on healthcare professionals opening conversations about death and dying. Whether EPaCCS facilitate such conversations is unclear.

We do not know if EPaCCS improve practice or simply document what is already taking place. Little research has been undertaken to understand how, and by whom, EPaCCS are being used and whether EPaCCS support patients' end of life wishes. Rigorous evaluation and research is needed to investigate to what extent EPaCCS influence services working together to support 'a good death'.

Aims Describe the socio-demographic characteristics of patients who die with an EPaCCS record, their cause and place of death and compare these with patients who die without an EPaCCS record. Explore the impact of an EPaCCS on the experience of receiving end of life care for patients and carers, and understand healthcare professionals' views and experiences of utilising an EPaCCS to provide coordinate care.

Methods This is a mixed-methods study, taking place in the Bristol, North Somerset and South Gloucestershire area, within a realist evaluation paradigm.

Results and conclusions Work in progress - data collection starting September 2018.

\section{P-57 MOVING FROM PAPER TO ELECTRONIC RECORD KEEPING WITHIN A CHILDREN'S HOSPICE}

Andrew Beaumont. Brian House Children's Hospice, Blackpool, UK

\subsection{6/bmjspcare-2018-hospiceabs.82}

The aim of the work was to enhance patient safety in the following ways:

- Ability to remotely access a patient record enabling advice or queries to be addressed without finding a set of case notes

- Reduced amount of telephone traffic between the multidisciplinary team and hospice staff around episodes of care enabling more timely and appropriate interventions to be offered to the children and their families 